\title{
The landscape of PDK1 in Breast Cancer
}

\section{Running title: PDK1 in breast cancer}

\author{
Na Wang ${ }^{1,2 *}$, Jianjiang $\mathrm{Fu}^{1,2 *}$, Zhihua $\mathrm{Li}^{1,2,3^{*}}$, Ningni Jiang ${ }^{1,2}$, Yanhong $\mathrm{Chen}^{1,2,4}$ and Juan \\ Peng ${ }^{1,2 \sharp}$
}

\author{
${ }^{1}$ Department of Pathology, Key Laboratory of Reproduction and Genetics of \\ Guangdong Higher Education Institutes, Key Laboratory for Major Obstetric \\ Diseases of Guangdong Province, The Third Affiliated Hospital of Guangzhou \\ Medical University, Guangzhou 510150, China; wangna10197@126.com (NW); \\ 2042632928@qq.com (JF); zhihuali2004@163.com (ZL); 649144805@qq.com (NJ); \\ 2011683122@gzhmu.edu.cn (YC) \\ 2 The Third Clinical School of Guangzhou Medical University, Guangzhou \\ 510150, China \\ ${ }^{3}$ Department of Fetal Medicine and Prenatal Diagnosis, The Third Affiliated \\ Hospital of Guangzhou Medical University, Guangzhou 510150, China \\ ${ }^{4}$ Department of Obstetrics and Gynecology, The Third Affiliated Hospital of \\ Guangzhou Medical University, Guangzhou 510150, China \\ *The first three authors contributed equally to this work. \\ * Correspondence: Correspondence: Juan Peng, M.D., Department of Pathology; The \\ Third Affiliated Hospital of Guangzhou Medical University, 63 Duobao \\ Road, Guangzhou, 510150, P. R. China; Telephone: +8620-81292969; Fax: \\ +8620-81292969; Email: pengjuan1979@yahoo.com; \\ pengjuan1979@gzhmu.edu.cn; ORCID: 0000-0001-6308-3040
}

\begin{abstract}
Given that 3-Phosphoinositide-dependent kinase 1 (PDK1) plays a crucial role in malignant biological behaviors of a wide-range of cancers, we further review the influence of PDK1 in breast cancer (BC). First, we describe the power of PDK1 in cellular behaviors and extensively demonstrate the interacting networks of PDK1 via PI3Kdependent/ PI3K-independent pathway. Then we enlighten the roles of PDK1 in carcinogenesis, growth and survival, metastasis, and chemoresistance in BC cells. More important, we sort the current preclinical or clinical trials of PDK1 targeted therapy in $B C$ and find that even though at present no selective PDK1 inhibitor is available for BC therapy, but the combination trials of PDK1 targeted therapy and other agents have demonstrated some benefit. Thus, there is increasing anticipations that PDK1 targeted therapy will have its space in future therapeutic concepts of $\mathrm{BC}$, and we hope to feature PDK1 in BC to the clinic and bring the new promising to patients for targeted therapies.
\end{abstract}

Keywords: PDK1; breast cancer; survival; prognosis; targeted therapy

\begin{abstract}
Abbreviations
AMIGO2, adhesion molecule with IgG-like domain 2; BC, breast cancer; BLBC, basal-like breast cancer; CNS, central nervous system; $\mathrm{CPT}$, camptothecin; $\mathrm{DCP}$, Des-gamma-carboxyl prothrombin; ER, estrogen receptor; HCC, hepatocellular carcinoma; HER2, human epidermal receptor 2; HM, hydrophobic motif; HMEC, human mammary epithelial cells; IBC, invasive breast carcinoma; IDC, invasive ductal carcinoma;
\end{abstract}


ICIs, immune checkpoint inhibitors; MTD, maximum tolerated dose; mTORC2, rictor-mTOR complex; NOS, not otherwise specified; NES, nuclear export signal; NST, no special type; PDK1, 3-phosphoinositide-dependent protein kinase 1; $\mathrm{PH}$, Pleckstrin homology; PI3K, phosphatidylinositol 3-kinase; PIF, PDK1-interacting fragment; PIP3, PtdIns $(3,4,5) \mathrm{P} 3$; PKC $\alpha$, protein kinase $\mathrm{C} \alpha$; PR, progesterone receptor; PRK2, protein kinase Crelated kinase-2; RCC, renal cell carcinoma; SCID, severe combined immunodeficient; SE, side effects; TME, tumor microenvironment; $\mathrm{TM} / \mathrm{Z}$, turn motif or zipper site; TNBC, triple negative breast cancer; Tyr, tyrosine; WHO, World Health Organization. 


\section{Introduction}

An estimated 19,292,789 new cancer cases and 9,958,133 cancer related deaths occurred in 2020 worldwidely with the increasing global cancer burden. Apparently, breast cancer $(11.7 \%$ of the total new cases, BC) had taken the place of lung cancer $(11.4 \%, \mathrm{LC})$ for the first time and become the most frequently occurring cancer worldwide. Meanwhile, it ranked the 5 th among causes for human cancer related death worldly in 2020 (684996 deaths, 6.9\%) (World Health Organization. Cancer Today. 2020; https://gco.iarc.fr/). In China, BC ranked the 4th (416,371 new cases, 9.1\%) out of the top 10 most frequently occurring cancer and 7 th cancer related death $(117,174$ deaths, $3.9 \%$ ) respectively in this globally recognised list in 2020 . In the 2019 World Health Organization (WHO) Classification of Breast Tumours, BC constitutes a series of heterogeneous groups of tumors with marked variation in clinical presentation, biological behavior, and response to therapy, in which invasive breast carcinoma (IBC) of no special type (NST) (Related terminology: IBC, not otherwise specified (NOS) or invasive ductal carcinoma (IDC)) is the largest group [1]. With the development and progression of molecular assay, the classification and management of BC are not only based primarily on clinicopathologic characteristics (morphology, size, grade, nodal status, etc.) and immunophenotypic profile (principally, estrogen receptor (ER), progesterone receptor (PR) and human epidermal receptor 2 (HER2)), but also depended on molecular classification (HER2 amplifications or mutations, ESR1 mutations, CDH1 somatic mutations, BRCA1 and BRCA2 mutations, GATA3 somatic mutations, TP53 somatic and germline mutations, PIK3CA and MAP3K1 somatic mutations, ETV6-NTRK3 and MYB-NFIB gene rearrangements, etc.) [1-2] . Cumulative data indicates 3-phosphoinositide-dependent protein kinase 1 (PDK1), which is a major kinase of the AGC protein kinase family, plays crucial roles in various cellular process via PI3K/AKT signaling in BC cells, including carcinogenesis, proliferation, survival, metastasis, tumor microenvironment (TME) regulation and drug resistance. Herein, we review the remarkably short but substantial history of PDK1 in BC to improve options of individualized therapy for patients with BCs.

\section{The PDK1 protein and PDK1 signaling pathway}

Since the term "AGC kinase" was coined by Hanks etc. in 1995 [3] (shown in Figure 1), AGC kinase family has attracted great attention to its therapeutic potentials in clinic [4]. The AGC kinase family contains at least 60 members among 21 subfamilies (AKT, CRIK, DMPK, GRK, LATS, MAST, MRCK, MSK, NDR, PDK1, PKA, PKC, PKG, PKN, PRK, ROCK, RSK, S6K, SGK, SGK494 and YANK), which share structural similarity and a common mechanism of activation concerning phosphorylation of two highly conserved motifs: the activation segment (referred to as the T-or activation loop) located in the catalytic kinase domain and the hydrophobic motif (HM) located in a non-catalytic region 
[5]. Some AGC kinases also possess a third phosphorylation site (the turn motif or zipper site, TM/Z), which promotes their integrity both by stabilizing the HM in its kinase-activating binding site and by protecting the HM from dephosphorylation [6].

As a member of AGC kinase family, PDK1 was initially discovered in rabbit skeletal muscle in 1997 [7], and then identified in human 293 cells [8], meanwhile, verified to be encoded by human PDK1 (aliases: PDPK1, PDPK2, PDPK2P, PRO0461; gene ID: 5170; located at 16p13.3; including 17 exons; with a full-length sequence of around $65 \mathrm{kDa}$; https://www.ncbi.nlm.nih.gov/gene) in 1997 [8]. Being a soluble and globular medium-sized kinase, PDK1 is a cytosolic protein of 556 amino acids composed of two well-characterized functional domains: the N-terminal catalytic kinase domain (the $\mathrm{T}$ - or activation loop) and the C-terminal Pleckstrin homology (PH) domain. Comparatively, PDK1 doesn't contain any HM, but displays a unique PDK1-interacting fragment (PIF) pocket in kinase domain, which is separate from the ATP- and substratebinding sites in kinase domain. Hence, PDK1 was unambiguously identified to not only be responsible for activating AKT by phosphorylating Thr308 T-loop residue, as well as being the major T-loop kinase for other AGC kinases, such as S6K, RSK, SGK and many PKC isoforms, but also interact with the PIF of protein kinase C-related kinase-2 (PRK2) through the PIF pocket, which serves as a "docking site" in PDK1 and enhances the phosphorylation of its substrates [9-10]. Interestingly, a second hydrophobic PIF pocket in kinase domain of PDK1 was also observed to mediate the interaction and phosphorylation of S6K1 by promoting the binding of S6K1 with PDK1 [11]. On the other hand, as the membrane targeting site of PDK1, PH domain was verified to not only specifically interact with the second messenger PtdIns(3,4,5)P3 (PIP3) and PtdIns(3,4)P2, which are the products of PI3K, but also directly bind a novel scaffold protein, adhesion molecule with IgG-like domain 2 (AMIGO2) at residues 465-474 [12-13].

Generally, there are two patterns involving PDK1 activation in cellular behaviors $[14,15]$. Classically, the increased PIP3 stimulated by growth factors or hormones switches on the catalytic activity of PDK1, which is kept under control by limiting its access to substrates in the absence of stimuli, in an inducible manner by aforementioned PIP3 binding, and then PDK1 is capable of phosphorylating and activating substrates, for example, PDK1-mediated AKT activation. Due to the PH domain possessed by both PDK1 and AKT, simultaneous PDK1 and AKT selectively binding with PIP3 brings double influence on fostering the interaction between the two kinases and determining a conformational change in AKT and PDK1, making AKT more prone to be phosphorylated on Thr308 of the T-loop by PDK1, which is regulated by the rictor-mTOR complex (mTORC2) via phosphorylating AKT on Ser473 of the HM [1618]. This process is referred to as the canonical PIP3/AKTdependent manner of PDK1 activation (shown in Figure 2). 
Alternatively, PDK1 can be activated in various noncanonical manners. In this setting, the afore-mentioned observations have indicated that the PIF pockets of PDK1 are mainly responsible for the phosphorylation of substrates (PRK2 and S6K1), which are lack of PH domain [10, 11]. Intriguingly, the facts that PDK1 phosphorylated and activated p70 S6 kinase at Thr252 [19], SGK at Ser422 [20] and p90 RSK at Ser227 [21] respectively, which were verified to be inactive by disrupting the PIF pocket of PDK1 in embryonic stem cells [22] have further illustrated that PDK1 activity could be monitored in such a PIP3independent manner. Growing evidence indicates PDK1 can also be phosphorylated on tyrosine (Tyr) residues including Tyr9/373/376 phosphorylation sites, resulting in an increase in activity. In fact, PDK1 activity was regulated by peroxovanadate through all three Tyr-9/373/376 phosphorylated, or by reversible tyrosine phosphorylation on Tyr-373/376 rather than Tyr-9 phosphorylation sites, or by Pyk2 in response to angiotensin II, which yielded to the sequential tyrosine phosphorylation on Tyr9 and Tyr-373/376 [23-25]. What is more, AMIGO2 as aforementioned directly interacts with PH domain of PDK1 in a non-PIP3-dependent fashion to govern cell survival and angiogenesis via activating the AKT pathway [13]. When extreme, PDK1 was observed to induce self-activation through trans-autophosphorylation on Ser241 of the T-loop constitutively [26].

\section{Potential roles of PDK1 translocation in BC cells}

In view of the ubiquitous expression of PDK1 in cells, even in fungi and plants, the uninterrupted presence of this kinase in such a large group of living organisms across long-term evolution manifests PDK1 is a key player in development and function of eukaryotic life. There is increasingly emerging evidence demonstrating that PDK1, which contains a hydrophobic nuclear output sequence, not only can typically translocate from the cytoplasm to plasma membrane (termed as membrane localization) with functional activation [13, 27], but also can translocate from the cytoplasm to nucleus, or vice versa, via a functional nuclear export signal (NES) stimulated by IGF-1 or PTEN loss. Videlicet, PDK1 is a cytoplasmic-nuclear-shuttling protein, and the fact that PDK-1 nuclear retention is blocked by the PI3K inhibitors suggests the cytoplasmic-nuclear-shuttling process of PDK-1 may be in a PI3K-dependent fashion [28, 29]. Moreover, nuclear PDK1 colocalizing with AKT and inhibiting FOXO3a transcriptional activity and nuclear localization [29] was further proved to increase nuclear pAKT abundance and suppress FOXO3a-dependent transcription of the gene encoding p27Kip1 via antiapoptotic signaling, as well as exhibit excellent potentiation of cell growth and proliferation [30]. Combined with these results and another discovery that in human prostate tumors, cytoplasm-localized PDK1 was associated with early low-risk tumors, while nuclear-localized PDK1 was associated with high tumor staging, the presence of solid tumor formation in mice induced by cells with nuclearlocalized PDK1 indicated that nuclear translocation of PDK1 
mediated the oncogenesis and tumor progression [30]. In resting human BC MDA-MB-231 cells, the inactive PDK1 and AKT were mainly distributed in the cytoplasm. PDK1 was observed to translocate from the cytosol region to plasma membrane in MDA-MB-231 cells in the presence of EGF, accompanied with the translocation and colocalization of $\mathrm{AKT}$ and $\mathrm{PKC} \zeta$ in the plasma membrane [31]. Furthermore, the number of BC cells, in which EGF-induced AKT translocation occurred, significantly reduced by knockdown of PDK1. Meanwhile, a decreased and delayed PKC $\zeta$ phosphorylation was detected by knockdown of PDK1 in these EGF stimulated BC cells compared with control cells [31]. Moreover, knockdown of PDK1 impaired EGF-induced cells adhesion and actin polymerization in BC cells, as well as tumorigenesis and lung colonization of BC cells in SCID mice. These results raised the possibility that PDK1 might mediate the migration and experimental metastasis of human BC cells [31]. Additionally, recent data showed that prolonged treatment of luminal BC cells with AKT inhibitors elicited FOXO3a dephosphorylation, nuclear translocation, and impaired its association with SirT6, eventually yielded to acetylated FOXO3a, which recognized the BD2 domain of BRD4, recruited the BRD4/RNAPII complex to the CDK6 gene promoter, and induced its transcription. And chemical interference with either BRD4/FOXO3a association or CDK6 significantly rescued the sensitivity of luminal BC cells to AKT inhibitors in vitro and in vivo [32]. Unfortunately, it is as yet unclear if or how nuclearlocalized PDK1 may affect the developmental or treatment events of $\mathrm{BC}$ cells by regulating AKT and FOXO3a. Therefore, more comprehensive study may conduce to exploring the potential roles of nuclear-localized PDK1 in BC cells via the PI3K/AKT pathway.

\section{Hyperactive PDK1 potentiates carcinogenesis by mediating transformation of mammary epithelial cells}

It is well established that the disruption of the normal autoinhibitory constraints on kinase activity involved in regulating cell proliferation and survival could lead to the malignant transformation of cells [33]. As a mediator of multiple signaling pathways coupled to growth factor receptor activation, the oncogenic role of PDK1 was evaluated in COMMA-1D mouse mammary epithelial cells. PDK1-expressing cells exhibited a high degree of transformation that was associated with the activation of AKT1 and elevated expression of protein kinase C $\alpha(\mathrm{PKC} \alpha)$. Cells overexpressing AKT1 did not exhibit anchorageindependent growth, whereas PKC $\alpha$ overexpression produced significant transformation, although to a lesser extent compared with PDK1. Coexpression of AKT1 and PKC $\alpha$ led to a more than additive effect on transformation activity. Isografts of either PDK1- or PKC $\alpha$-expressing cells but not AKT1-expressing cells in syngeneic mice led to formation of poorly differentiated mammary carcinomas. PDK1 was highly expressed in a majority of human BC cell lines [34]. These results were suggestive of PDK1-mediated transformation of mammary epithelial cells via PKC $\alpha$, but not AKT1 downstream. An oncogenic pathway 
downstream of PDK1 and PKC $\alpha$ that is associated with increased c-Myc and cyclin D1 expression through $\beta$-catenin activation, as well as activation of the PKC promoter and suppression of caveolin-1 expression was further confirmed in the follow-up research [35]. In fact, although the data that PDK1 expression was moderate to strong in BC specimens (213/241, 88\%, IHC) coincided with the findings in BC cells [34, 36], but PDK1 overexpression was not correlated with the mutation status of PIK3CA in BC specimens, which suggested that PDK1 could be independently activated in $\mathrm{BC}$, not just a part of the PIK3CA signaling pathway [36]. In agreement with these findings, Maurer et al. showed overexpression of total PDK1 protein (50/69, 72\%, IHC) and mRNA (RT-PCR), as well as PDK1 gene copy number increasing $(27 / 129,21 \%, \mathrm{FISH})$ in human BC samples [37]. Of note, PDK1 copy number increasing was associated with PIK3CA mutation, as well as patient survival [37]. More importantly, to determine the biological effects of PDK1 overexpression on ERBB2-induced transformation in vitro and tumor growth in vivo, a set of four human mammary epithelial cell lines MCF10A were generated by infected with retroviral vectors expressing PDK1 (+PDK1), the gene of the activated mutant rat homolog of ERBB2 (+NeuT), both (+PDK1+NeuT), or empty vector controls. The results that multiacinar structures were distorted and cell foci were linked by interconnecting branching tracts in +PDK1+NeuT MCF10A cells, which rapidly produced large muscle invasive tumors in all severe combined immunodeficient (SCID) mice indicated that increased PDK1 promoted ERBB2-induced transformation and tumor growth [37]. Similarly, the activated PDK1 displayed an especially important role either in carcinogenesis of MCF10A cells induced by the concurrent mutations in both KRAS and PIK3CA [38], or for the process of HRG/ERBB2-induced enhanced transformation of BC cells (SKBR3, MCF7 and ZR-75-1 cells) via AKT/TSC2/mTOR [39]. Noteworthily, PDK1 was observed to promote MYC-driven oncogenic transformation of not only HEK cells, but also immortalized human mammary epithelial cells (HMEC) and prostate epithelial cells via PDK1/PLK1/MYC signaling [40]. Thus, the evidence supporting the conclusion that hyperactive PDK1 potentiates mammary carcinogenesis by mediating transformation of mammary epithelial cells has been sufficient underlying these diverse views.

\section{Dysfunctional PDK1 signaling promotes cancer growth and survival of BC cells}

As the backbone of cancer cells biological events, cancer growth and survival have been extensively confirmed to be related to dysfunctional PDK1 signaling in various cancers, such as renal cell carcinoma (RCC) cells [41], hepatocellular carcinoma (HCC) cells [42], human glioblastoma cells [43, 44], melanoma and colon cancer cells [45]. The facts that PDK1 silencing reduced proliferation in MCF7 and T47D cells harboring PIK3CA mutation [37], as well as both genetic and chemical inhibition of PDK1 was sufficient to reduce soft agar colony formation of a panel of BC cell lines [46] had suggested a role for PDK1 in 
anchorage-independent cell growth. Furthermore, PDK1 was proved to be required for anchorage-independent and xenograft growth of BC cells (MDA-MB-231 and T47D cells) harboring either PI3KCA or KRAS mutations attributed to the facts that PDK1 knockdown led to increased anoikis, reduced soft agar growth, and pronounced apoptosis inside tumors, which could be reverted by PDK1 wild-type but not kinase-dead mutant, whereas could not be rescued by the expression of constitutively active forms of AKT in PDK1 silencing cells. Moreover, neither genetic nor pharmacological inhibition of AKT could suppress the effects of PDK1 overexpression [47]. In addition, enhanced PDK1 expression correlated with excessive metabolic activation in a spontaneous PyMT-induced BC mice model, and either pharmacological or genetic inhibition of PDK1 overwhelmed BC cell growth in vitro [48]. Intriguingly, a study reported that light at night activated IGF-1R/PDK1 signaling and accelerated tumor growth of MCF-7 cells in nude rats, which could be inhibited by PDK1 knockdown [49].

Taken together, these findings suggest that PDK1 contributes to cancer growth and survival of BC cells, even in the absence of PI3K oncogenic mutations, in either AKT-dependent or AKT-independent fashion, which makes PDK1 identified as a potential therapeutic target for $\mathrm{BC}$. However, the above evidence seems to be less convincing than the evidence coming from the following investigations of the role of PDK1 in BC metastasis to support that PDK1 is a potential therapeutic target in BC owing to the evidence that a small portion of PDK1-deficient tumorigenic cells eventually established BC lesions, albeit at a relatively later phase [48], as well as the evidence that PDK1 controlled migration and malignant transformation rather than cell growth and proliferation in PTEN-loss lymphocytes [50]. Further study is warranted to confirm the limited information on this topic in BC cells.

\section{Dysregulated PDK1 signaling facilitates metastasis of BC cells}

Metastasis being the leading cause of BC-related deaths worldwide means the multistage process of $\mathrm{BC}$ cells propagating from a primary site to progressively colonize distant organs through blood vessels/lymphatic vessels, including 4 steps: detachment, migration, invasion and adhesion [51-53]. Evidently, this complex succession of a spectrum of cellbiological events termed the "invasion-metastasis cascade" is closely related to tumor microenvironment (TME, consisting of extracellular matrix structure, growth factors, chemokines, matrix metalloproteinases, immune cells, etc.) regulation [51]. Recent decades have witnessed the molecular understanding of the mechanisms of dysregulated PDK1 signaling promoting BC metastasis.

Firstly, elevated phosphorylation and activation of PDK1pSer241 were observed not only in BC cell lines (non-invasive MCF-7 and invasive MDA-MBB-468 cells, WB), but also in invasive $\mathrm{BCs}(72 / 89,80.9 \%$, IHC), particularly in high-grade metastatic BCs $(18 / 21,86 \%$, IHC) [54]. Consistent findings were obtained as $90 \%$ of all tumor samples exhibited moderate to 
strong staining for PDK1pSer241, with $42 \%$ of evaluable samples exhibiting strong expression (74/177 cores, IHC) in tissue microarray analysis of human invasive BC [55]. Moreover, enhanced invasion on matrigel in PDK1-expressing Comma-1D mouse mammary epithelial cells (Comma/PDK1 cells, which produced invasive adenocarcinomas in mammary fat pad isografts) was confirmed and accompanied by increased MMP-2 activity resulting from stabilization against proteasomal degradation, as well as accompanied by elevated levels of MT1MMP, which in turn generated active MMP-2 and modulated the ECM proteins decorin and collagen. As such, PDK1 serves as an important effector of cell growth and invasion in the transformed cells [55]. The findings that, as described in detail above, PDK1 overexpression potentiated ERBB2 induced transformation and migration of human MCF10A cells [37], as well as that PDK1 regulated EGF-induced chemotaxis and directional migration and invasion of MCF10A cells by binding and activating MRCK $\alpha$ in an AKT-independent manner [56] have provided further insight into the influence of PDK1 on metastasis of the transformed cells. As discussed earlier, PDK1 depletion in BC cells inhibited metastasis by reducing EGF-mediated chemotaxis and adhesion of tumor cells and aggregation of actin via dampening phosphorylation and translocation of AKT and PKC $\zeta$ in a kinase-dependent manner, as well as suppressed tumorigenesis and lung colonization in SCID mice [31]. Additional evidence that PDK1 regulated invadopodia formation of BC cells (MDA-MB-231, BT-549, and Hs578T cells) via $\mathrm{p} 110 \alpha / \mathrm{PDK} 1 / \mathrm{AKT}$ signaling supports that PDK1 is implicated in metastasis of $\mathrm{BC}$ cells in a kinase-dependent manner [57, 58]. Interestingly, depletion of PDK1 disrupted cortical actin organization and cell motility of MTLn3 cells (highly metastatic mouse BC cells) in vitro and in vivo by regulating ROCK1 in a novel AKT-independent mechanism, in which ROCK1 associated with RhoE in the absence of PDK1 [59]. In fact, PDK1 regulated EGF-induced PLC $\gamma 1$ activation of MDAMB-231 cells in a different AKT-independent manner, and the interaction PDK1-PLC $\gamma 1$ was proved to be important for cancer cell invasion [60]. PDK1/PLC $\gamma 1$ interaction related to migration and invasion of $\mathrm{BC}$ cells was further validated to be blocked by a small molecule inhibitor 2-O-Bn-InsP5 in the following study [61]. Remarkably, the GENEOM study (NCT00959556) included blood sample collection from 342 BC patients through 88 SNPs analysis of PI3K/AKT/ mTOR pathway genes (including: AKT1 (17 SNPs), AKT2 (4), FGFR1 (2), mTOR (7), PDK1 (4), PI3KR1 (11), PI3KCA (20), PTEN (17) and RPS6KB1 (6)) demonstrated not only the association between PI3KR1-rs706716 and central nervous system (CNS) metastasis by univariate analysis, but also the associations between AKT1-rs3803304, AKT2-rs3730050, PDK1rs11686903 and PI3KR1-rs706716 and CNS metastasis by multivariate analysis [62].

Of course, apart from the effect of PDK1 deletion on T cell lineage development $[63,64]$, PDK1 was further proved to overwhelm metastasis of $\mathrm{BC}$ by regulating the immune cells of TME in PyMT-induced BC mice model by mediating 
macrophage polarization [48, 65]. Myeloid-specific inactivation of PDK1 reprogrammed metabolism of tumor-infiltrating macrophages and stimulated M1 macrophage polarization by inhibiting mTOR pathway, meanwhile, retarded tumor growth and suppressed lung metastasis of BC model [65].

In summary, PDK1 is unequivocally identified as a therapeutic target for the role of PDK1 in BC metastasis.

\section{Aberrant PDK1 signaling reinforces drug resistance in BC}

Owing to its essential role in metastatic potential, transformation induction and cell fate determination, it is not surprising that aberrant PDK1 plays a part in drug resistance of $B C$. More and more findings have been documented that hyperactive PDK1 signaling reinforces BC chemoresistance in various means. Given the fact that the expression and activationspecific phosphorylation levels of PDK1 and AKT1 varied greatly among the BC cell lines, and the fact that the phosphorylation of PDK1 or AKT1 was related to the sensitive of BC cells to chemotherapy drugs, cells with high levels of Ser241phosphorylated PDK1 (MDA453 and SKBR3 cells) were shown to be more resistant to gemcitabine-induced apoptosis than cells expressing high levels of Ser473-phosphorylated AKT1 (MDA468 and MDA231 cells). Furthermore, loss of PDK1 sensitized MCF7 cells to gemcitabine-induced apoptosis more effectively than did AKT1 ablation in the same cells. Thus, much attention has focused on PDK1, which might be a superior alternative to AKT1 as a target for sensitizing BC cells to gemcitabine [66]. Analogously, PDK1 was affirmed to be a key modifier of acquired resistance to CDK4/6 inhibitors in ERpositive BC cells via PI3K/PDK1 pathway in both AKTdependent and AKT-independent manners, and the combination of CDK4/6 inhibitor ribociclib or palbociclib and PDK1 inhibitor GSK2334470 synergistically suppressed proliferation and increased apoptosis in several $\mathrm{ER}+\mathrm{BC}$ cell lines in vitro and in vivo [67]. Additionally, PI3K-PDK1-AKT ${ }^{300}$ signaling axis was reported to sustain the survival of lapatinib-resistant BC cells (rBT474) [68].

Since SGK1 was verified to has a role in BC cells resistance to AKT inhibitors under PDK1 regulation [69], the PDK1-SGK1 axis was further revealed to overcome AKT inhibition by activating mTORC1 via direct phosphorylation of TSC2 in BC cells resistant to PI3K $\alpha$ inhibitors, while PDK1 deletion resensitized BC cells to PI3K $\alpha$ inhibitors [70]. Indeed, aside from the fact that the PDK1 signaling pathway was confirmed as a strong determinant of sensitivity to tamoxifen [71], TCRP1 was observed to induce tamoxifen resistance by modulating the PDK1/SGK1 signaling pathway in MCF-7 cells [72]. We thereby inferred PDK1-SGK1 axis displayed an especially important role for an acquired resistance mechanism in BC cells, which make them promising candidates in targeted therapeutics of $\mathrm{BC}$.

As noted, PDK1 not just induced oncogenic transformation, but evoked CSC-like phenotype via PDK1-PLK1-MYC signaling in MDA-MB-231 cells, which led to resistance to mTOR-targeted therapy [40]. 
Collectively, as emerging data has started to uncover its underestimated impacts on drug resistance, there are increasing expectations that encouraging results of preclinical or clinical studies of targeted PDK1 could be achieved in chemoresistance $\mathrm{BC}$ cells, or even better.

\section{Targeted therapies of PDK1 signaling in BC}

Over the past three decades, numerous studies for the role of PDK1 in cancers have yielded important results that paved the way for the development of anti-PDK1 monoclonal antibodies and specific or nonspecific PDK1 inhibitors. But so far, no selective PDK1 inhibitor has been available for BC therapy. We listed the preclinical or clinical trials of PDK1 targeted therapy in BCs according to the groups in Table 1 [40,46, 47, 57, 60, 61, 67, 70, 73-81] and extracted the important findings.

UCN-01, the first identified Chk1 inhibitor, was hereafter recognized as a nonselective inhibitor of PDK1, which was proved to have a potential hydrogen-bonding residue at the position equivalent to Thr222 T-loop in PDK1 [82-84]. UCN-01 was initially observed to inhibit the growth of $5 \mathrm{BC}$ cell lines (MCF-7, MDA-MB453, SK-BR-3, H85787 and MDA-MB468) and induce cell cycle arrest in 1993 [75], and then the maximum tolerated dose (MTD) of UCN-01 was estimated in patients with refractory BCs from 1995 to 2002 (NCT00001444). Furthermore, the MTD and side effects (SE) of UCN-01 and irinotecan hydrochloride in patients with metastatic, unresectable or resistant BCs had been evaluated since 2001 (NCT00031681). Interestingly, UCN-01 was affirmed to potentiate camptothecin (CPT)-induced cytotoxicity in two human BC cell lines with mutated p53 gene via modulating CPT-activated S and G2 checkpoints, which indicated the potential usefulness of combining UCN-01 in topoisomerase I inhibitor-based drug therapy for the treatment of $\mathrm{BC}$ with a dysfunctional p53 gene [76]. Additionally, based on the beneficial combination therapy of UCN-01 and irinotecan in p53-deficient triple negative breast cancer (TNBC) in vivo with human-in-mouse tumor models [77], Ma etc. further executed a phase II clinical trial of combination therapy of UCN-01 and irinotecan in 25 patients with metastatic TNBC, and found that effective UCN-01 could enhance irinotecan induced apoptosis in TP53 mutant BCs (69 \% tumors were basal-like BCs, BLBCs), as well as demonstrated that TNBC was molecularly heterogeneous and TP53 mutant TNBC was associated with a particularly poor survival, indicating a need to stratify patient populations in clinical trials [78]. Surprisedly, a recent study disclosed that pre-treatment of both non-tumourbearing and MDA-MB-468 tumour-bearing mice with UCN-01 prior to bolus 5-FU yielded enhanced therapeutic efficacy with significantly decreased tumor volumes and increased survival [79]. In short, these results provided a foundation for future clinical trials of UCN-01 in TNBC.

In a patent published in 2010, GSK2334470 was originally described as a highly specific inhibitor of PDK1 [85], and then characterized as more specific than UCN-01 or BX-795 with details in 2011, in which GSK2334470 not only inhibited PDK1 
from activating full-length AKT1 in the presence of PtdIns(3,4,5)P3-containing lipid vesicles or a mutant of AKT1 lacking the $\mathrm{PH}$ domain, but also inhibited PDK1 from phosphorylating the PDKtide peptide substrate [86]. As mentioned above, PDK1 regulated EGF-induced PLC $\gamma 1$ activation of MDA-MB-231 cells, and treatment with GSK2334470 resulted in total abrogation of the EGF-induced intracellular calcium increase and inositol phosphates accumulation, as well as inhibition of PLC $\gamma 1$ Tyr783 phosphorylation and cell invasion in MDA-MB-231 cells [60]. What's more, the evidence that PDK1 deletion with GSK2334470 re-sensitized BC cells to PI3K $\alpha$ inhibitor BYL719 [70] and CDK4/6 inhibitors respectively [67] had been encouraging enough to merit further clinical trials of GSK2334470 in patients with metastatic or acquired resistant BCs.

Notwithstanding that a new antibody targeting extracellular PDK1 was patented in 2016 [87], the treatment with anti-PDK1 monoclonal antibodies in BC cells or patients has not been reported till now. However, the combination therapy trials of PDK1 targeted therapy and other agents have demonstrated some benefit. With emerging creations and synthesis of PDK1 inhibitors [88], current and future efforts are underway to explore more options of PDK1 targeted therapy in BCs.

\section{Discussion}

Evidently, as a key protein kinase at the crossroad of AGC signaling pathways, PDK1 is inevitably a promising target with multiple roles in BC, particularly for those tumors with aggressiveness, metastasization and chemoresistance. Despite numerous attempts have been made in BC therapy with candidate PDK1 tool compounds, it is still lacking definitive evidence whether specific inhibitors of PDK1 as monotherapy could achieve favorable results, which urges us to take actions to determine the vulnerabilities of therapeutic potential in specificPDK1 inhibitors. Fortunately, besides the promotion of PDK1 inhibitors invention and development, several studies also enlightened the therapeutic potential of PDK1 inhibitors in combination with other agents in BCs. However, the clinical benefit of PDK1 inhibitors in BCs is not impressive enough as the other PI3K/AKT signaling inhibitors at present. It is imperative to develop more innovative options of targeted PDK1 treatment strategy in BCs. As noted above, the involvement of PDK1 in T cell lineage development $[63,64]$ makes it possible that PDK1 targeted therapy is combined with immunotherapy in BCs, such as immune checkpoint inhibitors (ICIs) anti-PD1/PDL1 therapy. Clinical feasibility, safety and potential effectiveness of combining PDK1 inhibitors in anti-PD1/PDL1-based drug therapy in BCs deserve to be further verified, which might lead to breakthroughs in $\mathrm{BC}$ treatment. Accordingly, the view that drug combination therapies of PDK1 inhibitors demonstrates some clinical efficacy in BCs will likely be accepted gradually in the upcoming years.

It's generally accepted that most cancers metastasize to specific organs, a process defined as "organotropism" [89]. To 
help develop effective treatments for metastatic BC, it is vital to gain a deeper understanding of the mechanisms by which BC metastasizes, particularly, those underlying organotropism towards brain, bone, lungs and liver [90]. Especially luminal BCs tend to metastasize to bone while BLBCs display organotropism metastasis of lung. Great amounts of data have demonstrated that exosomal microRNAs mediate BC metastasis to preferential premetastatic niches: brain, lungs, and bone. For instance, miR181c, miR-19a, miR-503 and miR-105 are involved in the brain metastasis of BC [91-96], miR23b, miR940, miR20a-5p and miR127 are associated with the bone metastasis [97,98], while miR105, miR202, miR-200a-c and miR210 mediate BC metastasis to lung [97]. Regarding the fact that PDK1 promoted tumor cell migration and metastatic spread into the lungs through regulating EMT [48], a study revealed that miR-155 harnessed tumor growth of BC cells in vivo via PIK3R1-PDK1/AKT-FOXO3a pathway [99], which evoked us to hypothesize that PDK1 might take part in organotropism metastasis of BC. We believe it would develop a novel perspective for the molecular mechanism research as to PDK1 in BC metastasis.

Overall, PDK1, which was underestimated, nowadays is uncovered to possess the compelling influence on the biological behavior of BC cells, particularly in carcinogenesis, proliferation and survival, metastasis, and drug resistance. Targeted PDK1 therapy has achieved some benefit in BCs, and we hope to feature PDK1 in BC to the clinic and bring the new promising to patients for targeted therapies.

Author Contributions: Juan Peng designed the review, wrote and revised the manuscript with input from Na Wang, Jianjiang Fu, Zhihua Li, Ningni Jiang, Yanhong Chen and Juan Peng. The literature search was performed and analyzed by $\mathrm{Na}$ Wang, Jianjiang $\mathrm{Fu}$, Zhihua Li, Ningni Jiang, Yanhong Chen and Juan Peng. The figures of the review were mainly painted by Jianjiang Fu and Juan Peng. The major part of Table 1 was completed by Na Wang and Juan Peng. All authors have read and agreed to the published version of the manuscript.

Funding: This work was funded by the National Natural Science Foundation of China (No. 81602631), the Student' Laboratory Open Project of Guangzhou Medical University (No. C195015021), 2017 Elite Talents Training Plan of the Third Affiliated Hospital of Guangzhou Medical University (Juan Peng).

Informed Consent Statement: Not applicable.

Acknowledgments:

Conflicts of Interest: The authors declare no conflict of interest.

\section{Reference}

1. WHO Classification of Tumours Editorial Board. World Health Organization (WHO) Classification of Tumours. Breast Tumours, 5th ed.; International Agency for Research on Cancer (IARC): Lyon, France, 2019; 102-109.

2. Hoda, S.A.; Brogi, E.; Koerner, F.C.; Rosen, P.P. Rosen's Breast Pathology, 5th ed.; Wolters Kluwer: Mexico, 2021; 10257-10263.

3. Hanks, S.K.; Hunter, T. Protein kinases 6. The eukaryotic protein kinase superfamily: kinase (catalytic) domain structure and classification. FASEB J. 1995, 9, 576-596. 
4. Xue, G.; Hemmings, B.A. AGC kinases in cancer metastasis, immune checkpoint regulation and drug resistance. Semin Cancer Biol. 2018, 48, iii-iv.

5. Pearce, L.R.; Komander, D.; Alessi, D.R. The nuts and bolts of AGC protein kinases. Nat Rev Mol Cell Biol. 2010, 11, 9-22.

6. Hauge, C.; Antal, T.L.; Hirschberg, D.; Doehn, U.; Thorup, K.; Idrissova, L.; Hansen, K.; Jensen, O.N.; Jorgensen, T.J.; Biondi, R.M.; et al. Mechanism for activation of the growth factor- activated AGC kinases by turn motif phosphorylation. EMBO J. 2007, 26, 2251-2261.

7. Alessi, D.R.; James, S.R.; Downes, C.P.; Holmes, A.B.; Gaffney, P.R.; Reese, C.B.; Cohen, P. Characterization of a 3-phosphoinositide-dependent protein kinase which phosphorylates and activates protein kinase Balpha. Curr Biol. 1997, 7, 261-269.

8. Alessi, D.R.; Deak, M.; Casamayor, A.; Caudwell, F.B.; Morrice, N.; Norman, D.G.; Gaffney, P.; Reese, C.B.; MacDougall, C.N.; Harbison, D.; et al. 3-Phosphoinositide-dependent protein kinase-1 (PDK1): structural and functional homology with the Drosophila DSTPK61 kinase. Curr Biol. 1997, 7, 776-789.

9. Bayascas, J.R. PDK1: the major transducer of PI3-Kinase actions. Curr Top Microbiol Immunol. 2010, 346, 9-29.

10. Biondi, R.M.; Cheung, P.C.; Casamayor, A.; Deak, M.; Currie, R.A.; Alessi, D.R. Identification of a pocket in the PDK1 kinase domain that interacts with PIF and the C-terminal residues of PKA. EMBO J. 2000, 19, 979-988.

11. Biondi, R.M.; Komander, D.; Thomas, C.C.; Lizcano, J.M.; Deak, M.; Alessi, D.R.; van Aalten, D.M. High resolution crystal structure of the human PDK1 catalytic domain defines the regulatory phosphopeptide docking site. EMBO J. 2002, 21, 4219-4228.

12. Deak, M.; Casamayor, A.; Currie, R.A.; Downes, C.P.; Alessi, D.R. Characterisation of a plant 3phosphoinositide-dependent protein kinase-1 homologue which contains a pleckstrin homology domain. FEBS Lett. 1999, 451, 220-226.

13. Park, H.; Lee, S.; Shrestha, P.; Kim, J.; Park, J.A.; Ko, Y.; Ban, Y.H.; Park, D.Y.; Ha, S.J.; Koh, G.Y.; et al. AMIGO2, a novel membrane anchor of PDK1, controls cell survival and angiogenesis via Akt activation. J Cell Biol. 2015, 211, 619-637.

14. Mora, A.; Komander, D.; van Aalten, D.M.; Alessi, D.R. PDK1, the master regulator of AGC kinase signal transduction. Semin Cell Dev Biol. 2004, 15, 161-170.

15. Gagliardi, P.A.; Puliafito, A.; Primo, L. PDK1: At the crossroad of cancer signaling pathways. Semin Cancer Biol. 2018, 48, 27-35.

16. James, S.R.; Downes, C.P.; Gigg, R.; Grove, S.J.; Holmes, A.B.; Alessi, D.R. Specific binding of the Akt-1 protein kinase to phosphatidylinositol 3,4,5-trisphosphate without subsequent activation. Biochem J. 1996, 315, 709-713.

17. Milburn, C.C.; Deak, M.; Kelly, S.M.; Price, N.C.; Alessi, D.R.; Van Aalten, D.M. Binding of phosphatidylinositol 3,4,5-trisphosphate to the pleckstrin homology domain of protein kinase B induces a conformational change. Biochem J. 2003, 375, 531-538.

18. Sarbassov, D.D.; Guertin, D.A.; Ali, S.M.; Sabatini, D.M. Phosphorylation and regulation of Akt/PKB by the rictor-mTOR complex. Science. 2005, 307, 1098-1101.

19. Alessi, D.R.; Kozlowski, M.T.; Weng, Q.P.; Morrice, N.; Avruch, J. 3-Phosphoinositide-dependent protein kinase 1 (PDK1) phosphorylates and activates the p70 S6 kinase in vivo and in vitro. Curr Biol. 1998, 8, 69-81.

20. Park, J.; Leong, M.L.; Buse, P.; Maiyar, A.C.; Firestone, G.L.; Hemmings, B.A. Serum and glucocorticoid-inducible kinase (SGK) is a target of the PI 3-kinase-stimulated signaling pathway. EMBO J. 1999, 18, 3024-3033.

21. Jensen, C.J.; Buch, M.B.; Krag, T.O.; Hemmings, B.A.; Gammeltoft, S.; Frodin, M. 90-kDa ribosomal S6 kinase is phosphorylated and activated by 3-phosphoinositide-dependent protein kinase-1. J Biol Chem. 1999, 274, 27168-27176.

22. Collins, B.J; Deak, M.; Arthur, J.S.; Armit, L.J.; Alessi, D.R. In vivo role of the PIF-binding docking site of PDK1 defined by knock-in mutation. EMBO J. 2003, 22, 4202-4211.

23. Park, J.; Hill, M.M.; Hess, D.; Brazil, D.P.; Hofsteenge, J.; Hemmings, B.A. Identification of tyrosine phosphorylation sites on 3-phosphoinositide-dependent protein kinase-1 and their role in regulating kinase activity. J. Biol. Chem. 2001, 276, 37459-37471.

24. Grillo, S.; Grémeaux, T.; Casamayor, A.; Alessi, D.R.; Le Marchand-Brustel, Y.; Tanti, J.F. Peroxovanadate induces tyrosine phosphorylation of phosphoinositide-dependent protein kinase-1 potential involvement of src kinase. Eur. J. Biochem. 2000, 267, 6642-6649.

25. Taniyama, Y.; Weber, D.S.; Rocic, P.; Hilenski, L.; Akers, M.L.; Park, J.; Hemmings, B.A.; Alexander, R.W.; Griendling, K.K. PYK2- and SRC-dependent tyrosine phosphorylation of PDK1 regulates focal adhesions. Mol. Cell Biol. 2003, 23, 8019-8029. 
26. Casamayor, A.; Morrice, N.A.; Alessi, D.R. Phosphorylation of Ser-241 is essential for the activity of 3-phosphoinositide-dependent protein kinase-1: identification of five sites of phosphorylation in vivo. Biochem J. 1999, 342, 287-292.

27. Biondi, R.M. Phosphoinositide-dependent protein kinase 1, a sensor of protein conformation. Trends Biochem Sci. 2004, 29, 136-142.

28. Lim, M.A.; Kikani, C.K.; Wick, M.J.; Dong, L.Q. Nuclear translocation of 3'-phosphoinositidedependent protein kinase 1 (PDK-1): a potential regulatory mechanism for PDK-1 function. Proc Natl Acad Sci U S A. 2003, 100, 14006-14011.

29. Scheid, M.P.; Parsons, M.; Woodgett, J.R. Phosphoinositide-dependent phosphorylation of PDK1 regulates nuclear translocation. Mol Cell Biol. 2005, 25, 2347-2363.

30. Kikani, C.K.; Verona, E.V.; Ryu, J.; Shen, Y.; Ye, Q.; Zheng, L.; Qian, Z.; Sakaue, H.; Nakamura, K.; $\mathrm{Du}, \mathrm{J}$.; et al. Proliferative and antiapoptotic signaling stimulated by nuclear-localized PDK1 results in oncogenesis. Sci Signal. 2012, 5, ra80.

31. Liu, Y.; Wang, J.; Wu, M.; Wan, W.; Sun, R.; Yang, D.; Sun, X.; Ma, D.; Ying, G.; Zhang, N. Downregulation of 3-phosphoinositide-dependent protein kinase-1 levels inhibits migration and experimental metastasis of human breast cancer cells. Mol Cancer Res. 2009, 7, 944-954.

32. Liu, J.; Duan, Z.; Guo, W.; Zeng, L.; Wu, Y.; Chen, Y.; Tai, F.; Wang, Y.; Lin, Y.; Zhang, Q.; et al. Targeting the BRD4/FOXO3a/CDK6 axis sensitizes AKT inhibition in luminal breast cancer. Nat Commun. 2018, 9, 5200.

33. Blume-Jensen, P.; Hunter, T. Oncogenic kinase signalling. Nature. 2001, 411, 355-365.

34. Zeng, X.; Xu, H.; Glazer, R.I. Transformation of mammary epithelial cells by 3-phosphoinositidedependent protein kinase-1 (PDK1) is associated with the induction of protein kinase Calpha. Cancer Res. 2002, 62, 3538-3543.

35. Xie, Z.; Zeng, X.; Waldman, T.; Glazer, R.I. Transformation of mammary epithelial cells by 3phosphoinositide- dependent protein kinase-1 activates beta-catenin and c-Myc, and downregulates caveolin-1. Cancer Res. 2003, 63, 5370-5375.

36. Arsenic, R. Immunohistochemical analysis of PDK1 expression in breast cancer. Diagn Pathol. 2014, $9,82$.

37. Maurer, M.; Su, T.; Saal, L.H.; Koujak, S.; Hopkins, B.D.; Barkley, C.R.; Wu, J.; Nandula, S.; Dutta, B.; Xie, Y.; et al. 3-Phosphoinositide-dependent kinase 1 potentiates upstream lesions on the phosphatidylinositol 3-kinase pathway in breast carcinoma. Cancer Res. 2009, 69, 6299-6306.

38. Wang, G.M; Wong, H.Y.; Konishi, H.; Blair, B.G.; Abukhdeir, A.M.; Gustin, J.P.; Rosen, D.M.; Denmeade, S.R.; Rasheed, Z.; Matsui, W.; et al. Single copies of mutant KRAS and mutant PIK3CA cooperate in immortalized human epithelial cells to induce tumor formation. Cancer Res. 2013, 73, 3248-3261.

39. Lin, M.C.; Rojas, K.S.; Cerione, R.A.; Wilson, K.F. Identification of mTORC2 as a necessary component of HRG/ErbB2-dependent cellular transformation. Mol Cancer Res. 2014, 12, 940-952.

40. Tan, J.; Li, Z.; Lee, P.L.; Guan, P.; Aau, M.Y.; Lee, S.T.; Feng, M.; Lim, C.Z.; Lee, E.Y.; Wee, Z.N.; et al. PDK1 signaling toward PLK1-MYC activation confers oncogenic transformation, tumor-initiating cell activation, and resistance to mTOR-targeted therapy. Cancer Discov. 2013, 3, 1156-1171.

41. Zhou, W.M.; Wu, G.L.; Huang, J.; Li, J.G.; Hao, C.; He, Q.M.; Chen, X.D.; Wang, G.X.; Tu, X.H. Low expression of PDK1 inhibits renal cell carcinoma cell proliferation, migration, invasion and epithelial mesenchymal transition through inhibition of the PI3K-PDK1-AKT pathway. Cell Signal. 2019, 56, 114.

42. Liu, C.; Mu, X.; Wang, X.; Zhang, C.; Zhang, L.; Yu, B.; Sun, G. Ponatinib inhibits proliferation and induces apoptosis of liver cancer cells, but its efficacy is compromised by its activation on PDK1/Akt/mTOR signaling. Molecules. 2019, 24, 1363.

43. Flynn, P.; Wongdagger, M.; Zavar, M.; Dean, N.M.; Stokoe, D. Inhibition of PDK-1 activity causes a reduction in cell proliferation and survival. Curr Biol. 2000, 10, 1439-1442.

44. Luo, D.; Xu, X.; Li, J.; Chen, C.; Chen, W.; Wang, F.; Xie, Y.; Li, F. The PDK1/c-Jun pathway activated by TGF-beta induces EMT and promotes proliferation and invasion in human glioblastoma. Int $J$ Oncol. 2018, 53, 2067-2080.

45. Lu, Z.; Cox-Hipkin, M.A.; Windsor, W.T.; Boyapati, A. 3-phosphoinositide-dependent protein kinase-1 regulates proliferation and survival of cancer cells with an activated mitogen-activated protein kinase pathway. Mol Cancer Res. 2010, 8, 421-432.

46. Nagashima, K.; Shumway, S.D.; Sathyanarayanan, S.; Chen, A.H.; Dolinski, B.; Xu, Y.; Keilhack, H.; Nguyen, T.; Wiznerowicz, M.; Li, L.; et al. Genetic and pharmacological inhibition of PDK1 in cancer cells. J Biol Chem. 2011, 286, 6433-6448. 
47. Gagliardi, P.A.; di Blasio, L.; Orso, F.; Seano, G.; Sessa, R.; Taverna, D.; Bussolino, F.; Primo, L. 3Phosphoinositide-dependent kinase 1 controls breast tumor growth in a kinase-dependent but Aktindependent manner. Neoplasia. 2012, 14, 719-731.

48. Du, J.; Yang, M.; Chen, S.; Li, D.; Chang, Z.; Dong, Z. PDK1 promotes tumor growth and metastasis in a spontaneous breast cancer model. Oncogene. 2016, 35, 3314-3323.

49. Wu, J.; Dauchy, R.T.; Tirrell, P.C.; Wu, S.S.; Lynch, D.T.; Jitawatanarat, P.; Burrington, C.M.; Dauchy, E.M.; Blask, D.E.; Greene, M.W. Light at night activates IGF-1R/PDK1 signaling and accelerates tumor growth in human breast cancer xenografts. Cancer Res. 2011, 71, 2622-2631.

50. Finlay, D.K.; Sinclair, L.V.; Feijoo, C.; Waugh, C.M.; Hagenbeek, T.J.; Spits, H.; Cantrell, D.A. Phosphoinositide-dependent kinase 1 controls migration and malignant transformation but not cell growth and proliferation in PTEN-null lymphocytes. J Exp Med. 2009, 206, 2441-2454.

51. Guan, X. Cancer metastases: challenges and opportunities. Acta Pharm Sin B. 2015, 5, 402-418.

52. Steeg, P.S. Targeting metastasis. Nat Rev Cancer. 2016, 16, 201-218.

53. Menyailo, M.E.; Tretyakova, M.S; Denisov, E.V. Heterogeneity of circulating tumor cells in breast cancer: identifying metastatic seeds. Int J Mol Sci. 2020, 21, 1696.

54. Lin, H.J.; Hsieh, F.C.; Song, H.; Lin, J. Elevated phosphorylation and activation of PDK-1/AKT pathway in human breast cancer. Br J Cancer. 2005, 93, 1372-1381.

55. Xie, Z.; Yuan, H.; Yin, Y.; Zeng, X.; Bai, R.; Glazer, R.I. 3-phosphoinositide-dependent protein kinase1 (PDK1) promotes invasion and activation of matrix metalloproteinases. BMC Cancer. 2006, 6, 77.

56. Gagliardi, P.A.; di Blasio, L.; Puliafito, A.; Seano, G.; Sessa, R.; Chianale, F.; Leung, T; Bussolino, F.; Primo, L. PDK1-mediated activation of $\mathrm{MRCK} \alpha$ regulates directional cell migration and lamellipodia retraction. J Cell Biol. 2014, 206, 415-434.

57. Yamaguchi, H.; Yoshida, S.; Muroi, E.; Yoshida, N.; Kawamura, M.; Kouchi, Z.; Nakamura, Y.; Sakai, R.; Fukami, K. Phosphoinositide 3-kinase signaling pathway mediated by p110 $\alpha$ regulates invadopodia formation. J Cell Biol. 2011, 193,1275-1288.

58. Di Blasio, L.; Gagliardi, P.A.; Puliafito, A.; Primo, L. Serine/Threonine kinase 3-phosphoinositidedependent protein kinase-1 (PDK1) as a key regulator of cell migration and cancer dissemination. Cancers (Basel). 2017, 9, 25.

59. Pinner, S.; Sahai, E. PDK1 regulates cancer cell motility by antagonising inhibition of ROCK1 by RhoE. Nat Cell Biol. 2008, 10, 127-137.

60. Raimondi, C.; Chikh, A.; Wheeler, A.P.; Maffucci, T.; Falasca, M. A novel regulatory mechanism links PLC $\gamma 1$ to PDK1. J Cell Sci. 2012, 125, 3153-3163.

61. Raimondi, C.; Calleja, V.; Ferro, R.; Fantin, A.; Riley, A.M.; Potter, B.V.; Brennan, C.H.; Maffucci, T.; Larijani, B.; Falasca, M. A small molecule inhibitor of PDK1/PLC $\gamma 1$ interaction blocks breast and melanoma cancer cell invasion. Sci Rep. 2016, 6, 26142.

62. Le Rhun, E.; Bertrand, N.; Dumont, A.; Tresch, E.; Le Deley, M.C.; Mailliez, A.; Preusser, M.; Weller, M.; Revillion, F.; Bonneterre, J. Identification of single nucleotide polymorphisms of the PI3K-AKTmTOR pathway as a risk factor of central nervous system metastasis in metastatic breast cancer. Eur J Cancer. 2017, 87, 189-198.

63. Hinton, H.J.; Alessi, D.R.; Cantrell, D.A. The serine kinase phosphoinositide-dependent kinase 1 (PDK1) regulates T cell development. Nat Immunol. 2004, 5, 539-545.

64. Kelly, A.P.; Finlay, D.K.; Hinton, H.J.; Clarke, R.G.; Fiorini, E.; Radtke, F.; Cantrell, D.A. Notchinduced $\mathrm{T}$ cell development requires phosphoinositide-dependent kinase 1. EMBO J. 2007, 26, 34413450 .

65. He, Y.; Du, J.; Dong, Z. Myeloid deletion of phosphoinositide-dependent kinase-1 enhances NK cellmediated antitumor immunity by mediating macrophage polarization. Oncoimmunology. 2020, 9, 1774281.

66. Liang, K.; Lu, Y.; Li, X.; Zeng, X.; Glazer, R.I.; Mills, G.B.; Fan, Z. Differential roles of phosphoinositide-dependent protein kinase-1 and Akt1 expression and phosphorylation in breast cancer cell resistance to paclitaxel, doxorubicin, and gemcitabine. Mol Pharmacol. 2006, 70, 1045-1052.

67. Jansen, V.M.; Bhola, N.E.; Bauer, J.A.; Formisano, L.; Lee, K.M.; Hutchinson, K.E.; Witkiewicz, A.K.; Moore, P.D.; Estrada, M.V.; Sánchez, V.; et al. Kinome-wide RNA interference screen reveals a role for PDK1 in acquired resistance to CDK4/6 inhibition in ER-positive breast cancer. Cancer Res. 2017, 77, 2488-2499.

68. Xia, W.; Petricoin, EF 3rd.; Zhao, S.; Liu, L.; Osada, T.; Cheng, Q.; Wulfkuhle, J.D.; Gwin, W.R.; Yang, X.; Gallagher, R.I.; et al. An heregulin-EGFR-HER3 autocrine signaling axis can mediate acquired lapatinib resistance in HER2+ breast cancer models. Breast Cancer Res. 2013, 15, R85.

69. Sommer, EM.; Dry, H.; Cross, D.; Guichard, S.; Davies, B.R.; Alessi, D.R. Elevated SGK1 predicts resistance of breast cancer cells to Akt inhibitors. Biochem J. 2013, 452, 499-508. 
70. Castel, P.; Ellis, H.; Bago, R.; Toska, E.; Razavi, P.; Carmona, F.J.; Kannan, S.; Verma, C.S.; Dickler, M.; Chandarlapaty, S.; et al. PDK1-SGK1 signaling sustains AKT-independent mTORC1 activation and confers resistance to PI3K $\alpha$ inhibition. Cancer Cell. 2016, 30, 229-242.

71. Iorns, E.; Lord, C.J.; Ashworth, A. Parallel RNAi and compound screens identify the PDK1 pathway as a target for tamoxifen sensitization. Biochem J. 2009, 417, 361-370.

72. Zhao, S.; Li, X.; Yin, L.; Hou, L.; Lan, J.; Zhu, X. TCRP1 induces tamoxifen resistance by promoting the activation of SGK1 in MCF-7 cells. Oncol Rep. 2020, 43, 2017-2027.

73. Meuillet, E.J.; Zuohe, S.; Lemos, R.; Ihle, N.; Kingston, J.; Watkins, R.; Moses, S.A.; Zhang, S.; DuCuny, L.; Herbst, R.; et al. Molecular pharmacology and antitumor activity of PHT-427, a novel Akt/ phosphatidylinositide-dependent protein kinase 1 pleckstrin homology domain inhibitor. Mol Cancer Ther. 2010, 9, 706-717.

74. Arndt JC, T.; Guckian, K.; Kumaravel, G.; Lee, WC.; Lin, Edward Y.S.; Scott, D.; Sun, L.; Thomas, J.; Van Vloten, K.; Wang, D.; et al. Sunesis Pharmaceuticals, Inc.; Millennium Pharmaceuticals, Inc. Heterocyclic compounds useful as PDK1 inhibitors. 2018. AU2018222943.

75. Seynaeve, C.M.; Stetler-Stevenson, M.; Sebers, S.; Kaur, G.; Sausville, E.A.; Worland, P.J. Cell cycle arrest and growth inhibition by the protein kinase antagonist UCN-01 in human breast carcinoma cells. Cancer Res. 1993, 53, 2081-2086.

76. Jones, C.B.; Clements, M.K.; Wasi, S.; Daoud, S.S. Enhancement of camptothecin-induced cytotoxicity with UCN-01 in breast cancer cells: abrogation of S/G (2) arrest. Cancer Chemother Pharmacol. 2000, 45, 252-258.

77. Ma, C.X.; Cai, S.; Li, S.; Ryan, C.E.; Guo, Z.; Schaiff, W.T.; Lin, L.; Hoog, J.; Goiffon, R.J.; Prat, A.; et al. Targeting Chk1 in p53-deficient triple-negative breast cancer is therapeutically beneficial in human-in-mouse tumor models. J Clin Invest. 2012, 122, 1541-1552.

78. Ma, C.X.; Ellis, M.J.; Petroni, G.R.; Guo, Z.; Cai, S.R.; Ryan, C.E.; Craig Lockhart, A.; Naughton, M.J.; Pluard, T.J.; Brenin, C.M.; et al. A phase II study of UCN-01 in combination with irinotecan in patients with metastatic triple negative breast cancer. Breast Cancer Res Treat. 2013, 137, 483-492.

79. Mull, B.B.; Livingston, J.A.; Patel, N.; Bui, T.; Hunt, K.K.; Keyomarsi, K. Specific, reversible G1 arrest by UCN-01 in vivo provides cytostatic protection of normal cells against cytotoxic chemotherapy in breast cancer. Br J Cancer. 2020, 122, 812-822.

80. Tseng, P.H.; Wang, Y.C.; Weng, S.C.; Weng, J.R.; Chen, C.S.; Brueggemeier, R.W.; Shapiro, C.L.; Chen, C.Y.; Dunn, S.E.; Pollak, M.; et al. Overcoming trastuzumab resistance in HER2-overexpressing breast cancer cells by using a novel celecoxib-derived phosphoinositide-dependent kinase-1 inhibitor. Mol Pharmacol. 2006, 70, 1534-1541.

81. Boehringer Ingelheim International Gmbh. Pyridons as PDK1 inhibitors. 2011. US0269958.

82. Takahashi, I.: Kobayashi, E.; Asano, K.; Yoshida, M.; Nakano, H. UCN-01, a selective inhibitor of protein kinase C from Streptomyces. J Antibiot (Tokyo). 1987, 40, 1782-1784.

83. Komander, D.; Kular, G.S.; Bain, J; Elliott, M.; Alessi, D.R.; Van Aalten, D.M. Structural basis for UCN-01 (7-hydroxystaurosporine) specificity and PDK1 (3-phosphoinositide-dependent protein kinase-1) inhibition. Biochem J. 2003, 375, 255-262.

84. Alcántara-Hernández, R.; Hernández-Méndez, A.; García-Sáinz, J.A. The phosphoinositidedependent protein kinase 1 inhibitor, UCN-01, induces fragmentation: possible role of metalloproteinases. Eur J Pharmacol. 2014, 740, 88-96.

85. Axten, J.M.; Blackledge, C.W.; Brady, G.P.; Feng, Y.; Grant, S.W.; Medina, J.R.; Milller, W.H.; Romeril, S.P. Preparation of 6-(4-pyrimidinyl)- 1H-indazole derivatives as PDK1 inhibitors. 2010. PCT/US2009/064891, WO 2010059658.

86. Najafov, A.; Sommer, E.M.; Axten, J.M.; Deyoung, M.P.; Alessi, D.R. Characterization of GSK2334470, a novel and highly specific inhibitor of PDK1. Biochem. J. 2011, 433, 357-369.

87. Dessain S. K.; B.C. H, inventors; Dessain S. K.; Heimbach B.C. PDK1 binding molecules and uses thereof. 2016. US2016319035 (A1).

88. Sestito. S.; Rapposelli, S. A patent update on PDK1 inhibitors (2015-present). Expert Opin Ther Pat. 2019, 29, 271-282.

89. Gao, Y.; Bado, I.; Wang, H.; Zhang, W.; Rosen, J.M.; Zhang, X.H. Metastasis organotropism: redefining the congenial soil. Dev Cell. 2019, 49, 375-391.

90. Van Mechelen, M.; Van Herck, A.; Punie, K.; Nevelsteen, I.; Smeets, A.; Neven, P.; Weltens, C.; Han, S.; Vanderstichele, A.; Floris, G.; et al. Behavior of metastatic breast cancer according to subtype. Breast Cancer Res. Treat. 2020, 181, 115-125.

91. Liu, Q.; Peng, F.; Chen, J. The Role of Exosomal MicroRNAs in the tumor microenvironment of breast cancer. Int. J. Mol. Sci. 2019, 20, 3884. 
92. Le, M.T.; Hamar, P.; Guo, C.; Basar, E.; Perdigao-Henriques, R.; Balaj, L.; Lieberman, J. miR-200containing extracellular vesicles promote breast cancer cell metastasis. J. Clin. Invest. 2014, 124, 51095128.

93. Ekici, K.; Temelli, O.; Dikilitas, M.; Halil Dursun, I.; Bozdag Kaplan, N.; Kekilli, E. Survival and prognostic factors in patients with brain metastasis: Single center experience. J. BUON. 2016, 21, 958963.

94. Zhou, W.; Fong, M.Y.; Min, Y.; Somlo, G.; Liu, L.; Palomares, M.R.; Yu, Y.; Chow, A.; O'Connor, S.T.; Chin, A.R.; et al. Cancer-secreted miR-105 destroys vascular endothelial barriers to promote metastasis. Cancer Cell. 2014, 25, 501-515.

95. Lee, T.H.; Avraham, H.K.; Jiang, S.; Avraham, S. Vascular endothelial growth factor modulates the transendothelial migration of MDA-MB-231 breast cancer cells through regulation of brain microvascular endothelial cell permeability. J. Biol. J Biol Chem. 2003, 278, 5277-5284.

96. Hashimoto, K.; Ochi, H.; Sunamura, S.; Kosaka, N.; Mabuchi, Y.; Fukuda, T.; Yao, K.; Kanda, H.; Ae, K.; Okawa, A.; et al. Cancer-secreted hsa-miR-940 induces an osteoblastic phenotype in the bone metastatic microenvironment via targeting ARHGAP1 and FAM134A. Proc. Natl. Acad. Sci. USA. 2018, 115, 2204-2209.

97. Guo, L.; Zhu, Y.; Li, L.; Zhou, S.; Yin, G.; Yu, G.; Cui, H. Breast cancer cell-derived exosomal miR20a-5p promotes the proliferation and dierentiation of osteoclasts by targeting SRCIN1. Cancer Med. 2019, 8, 5687-5701.

98. Jin, L.; Han, B.; Siegel, E.; Cui, Y.; Giuliano, A.; Cui, X. Breast cancer lung metastasis: molecular biology and therapeutic implications. Cancer Biol Ther. 2018, 19, 858-868.

99. Kim, S.; Lee, E.; Jung, J.; Lee, J.W.; Kim, H.J.; Kim, J.; Yoo, H.J.; Lee, H.J.; Chae, S.Y.; Jeon, S.M.; et al. microRNA-155 positively regulates glucose metabolism via PIK3R1-FOXO3a-cMYC axis in breast cancer. Oncogene. 2018, 37, 2982-2991.

Figure 1 Timeline of the hallmarks in PDK1 pathway research in breast cancer.

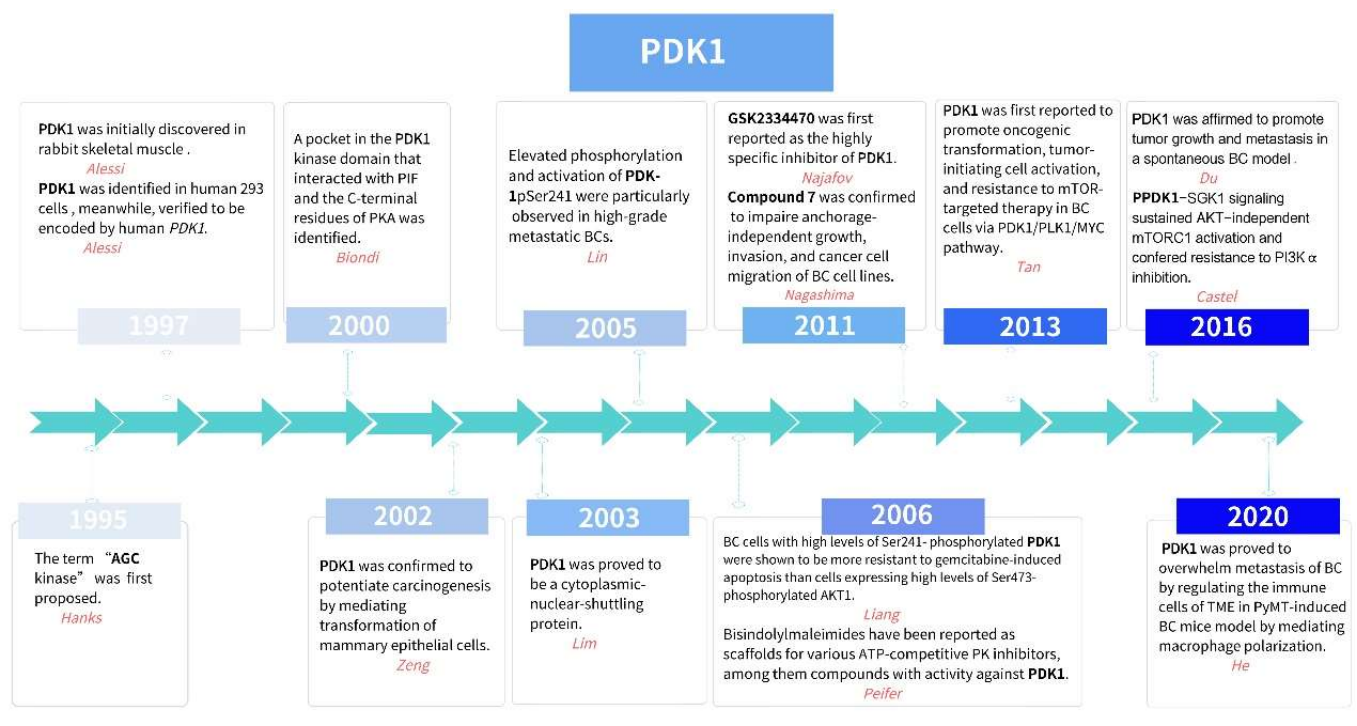


Figure 2 Overview of PDK1 signaling in breast cancer. The illustration of the molecule mechanism of PDK1 downstream signaling pathways and the crosstalk between PDK1 and other cell signal transduction pathways in BC. Classically, the increased PIP3 stimulated by growth factors or hormones switches on the catalytic activity of PDK1, then PDK1 is capable of phosphorylating and activating AKT. The simultaneous PDK1 and AKT selectively binding with PIP3 brings double influence on fostering the interaction between the two kinases and determining a conformational change in AKT and PDK1, making AKT more prone to be phosphorylated on Thr308 of the T-loop by PDK1, which is regulated by the rictormTOR complex (mTORC2) via phosphorylating AKT on Ser473 of the HM. This process is referred to as the canonical PIP3/AKT manner of PDK1 activation. Alternatively, PDK1 can be activated in various non-canonical manners. First, AMIGO2 interacted with PDK1 by directly binding with PH domain to govern cell survival and angiogenesis via activating the AKT pathway. PDK1 was proved to translocate from the cytoplasm to nucleus, or vice versa, via a functional nuclear export signal (NES) stimulated by IGF-1 or PTEN loss in the form of mPDK1 $1^{\triangle 382-391}$. Moreover, nuclear PDK1 colocalizing with AKT and inhibiting FOXO3a transcriptional activity and nuclear localization was further proved to increase nuclear pAKT abundance and suppress FOXO3a-dependent transcription of the gene encoding $\mathrm{p} 27^{\mathrm{Kip} 1}$ via antiapoptotic signaling, as well as exhibit excellent potentiation of cell growth and proliferation. Intriguingly, a study reported that light at night activated IGF-1R/PDK1 signaling and accelerated tumor growth of MCF-7 cells in nude rats, which could be inhibited by PDK1 knockdown. In addition, depletion of PDK1 disrupted cortical actin organization and cell motility of MTLn3 cells in vitro and in vivo by regulating ROCK1 in a novel AKT-independent mechanism, in which ROCK1 associated with RhoE in the absence of PDK1. Similarly, the activated PDK1 displayed an especially important role either in carcinogenesis of MCF10A cells induced by the concurrent mutations in both $K R A S$ and $P I K 3 C A$, or for the process of HRG/ERBB2-induced enhanced transformation of BC cells via AKT/TSC2/mTOR. 
Noteworthily, PDK1 was observed to promote MYC-driven oncogenic transformation of not only HEK cells, but also immortalized human mammary epithelial cells and prostate epithelial cells via PDK1/PLK1/MYC signaling. In fact, PDK1 regulated EGF-induced PLC $\gamma 1$ activation of MDA-MB-231 cells in a different AKTindependent manner, and the interaction PDK1-PLC $\gamma 1$ was proved to be important for cancer cell invasion. Finally, TCRP1 could induce tamoxifen resistance by regulating the PDK1/SGK1 signaling pathway.

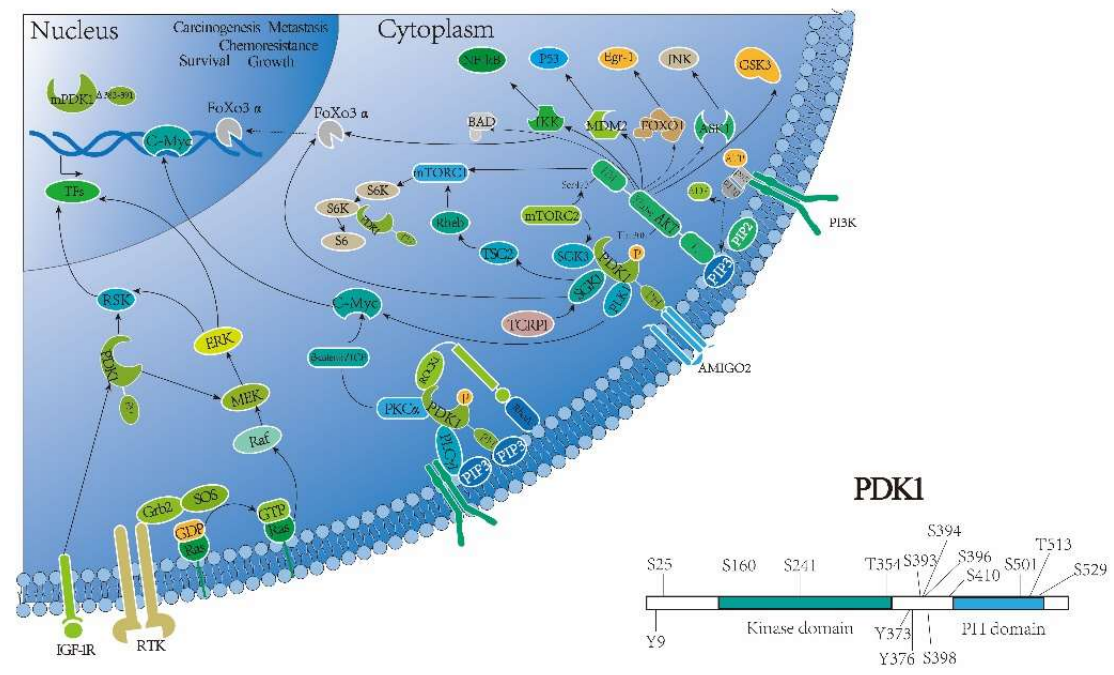


Table 1 Preclinical and clinical trials of PDK1 targeted therapies in breast cancers (as

of September 2021)

(http://clinicaltrials.gov)

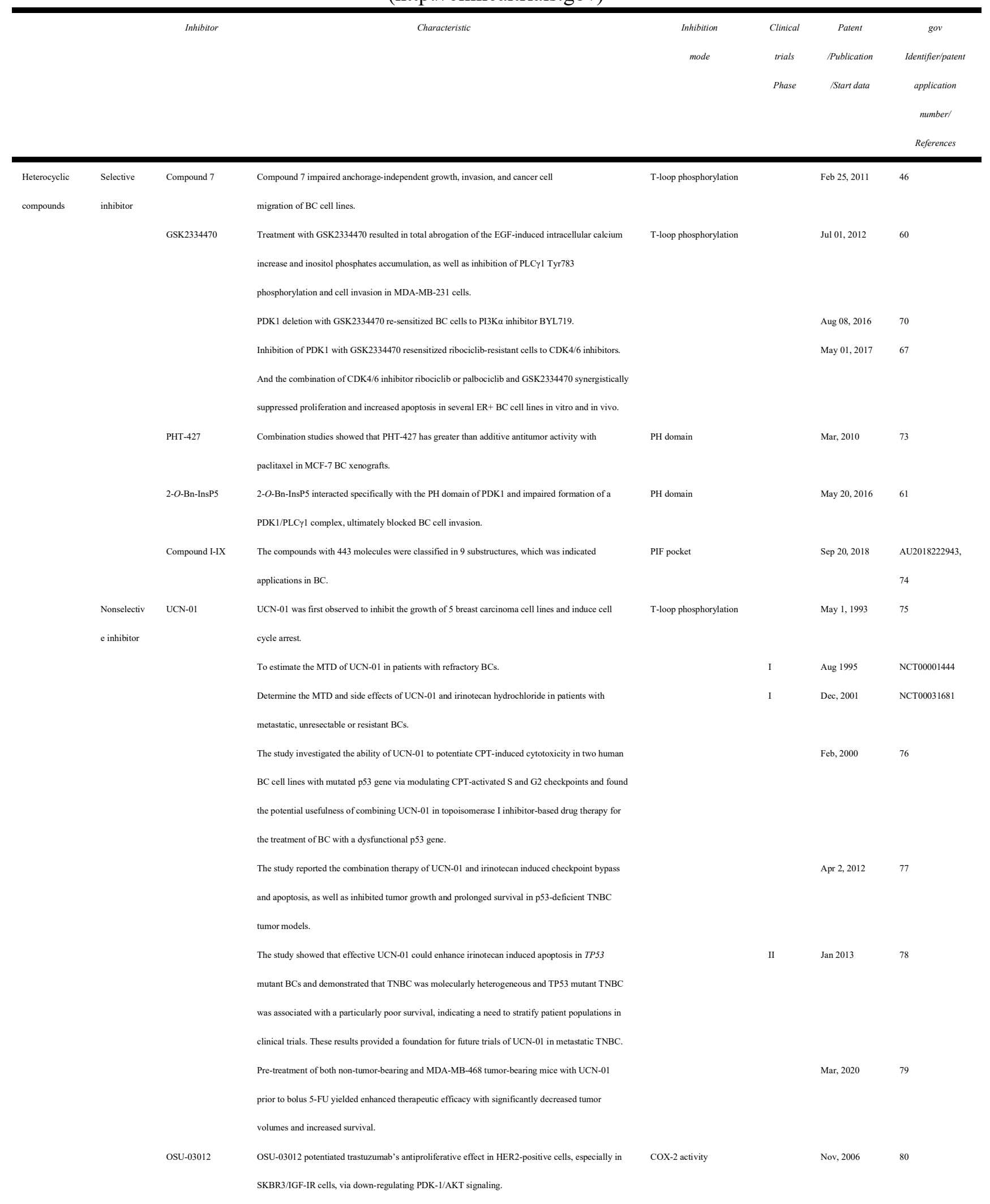




\begin{tabular}{|c|c|c|c|c|c|c|}
\hline & & & OSU-03012 blocked invadopodia formation of MDA-MB-231 cells. & & Jun 27,2011 & 57 \\
\hline & & & OSU-03012 blocked anchorage-independent growth of MDA-MB-231 cells and promoted anoikis. & & Aug, 2012 & 47 \\
\hline & & BX-795 & BX-795 inhibited soft agar growth of BC cells very effectively. & T-loop phosphorylation & Aug, 2012 & 47 \\
\hline & & & BX-795 treatment resulted in similar MYC depletion in all these cells but preferentially reduced the & & Oct, 2013 & 40 \\
\hline & & & cell viability of MYC-dependent breast cancer cell lines (MDA-MB-231, Hs578T, and SUM 159PT) & & & \\
\hline & & & as compared with the MYC-independent breast cancer cell lines (T47D and BT474). Moreover, & & & \\
\hline & & & BX795 treatment resulted in marked inhibition of tumorsphere formation in MDA-MB-231 cells. & & & \\
\hline & & BX-912 & BX-912 treatment resulted in similar MYC depletion in all these cells but preferentially reduced the & T-loop phosphorylation & Oct, 2013 & 40 \\
\hline & & & cell viability of MYC-dependent breast cancer cell lines (MDA-MB-231, Hs578T, and SUM159PT) & & & \\
\hline & & & as compared with the MYC-independent breast cancer cell lines (T47D and BT474). Moreover, & & & \\
\hline & & & BX912 treatment resulted in marked inhibition of tumorsphere formation in MDA-MB-231 cells. & & & \\
\hline Pyridonyl & Nonselectiv & Pyridons & Pyridons was found to be effective against MDA-MB-468 cells, where the PI3K/AKT/PDK1 & & Nov, 2011 & US0269958, 81 \\
\hline derivatives & e inhibitor & & signaling pathway is up-regulated. & & & \\
\hline & & Pyridons & Pyridons were tested for cell growth/death in several human BC cell lines (BT474, HCC1954, T- & & 2010 & wo019637 \\
\hline & & & DD). There were no reports on in vivo data or selectivity, despite their potent in vitro activity. & & & \\
\hline
\end{tabular}

